\title{
On asymptotic dimension of amalgamated products and right-angled Coxeter groups
}

\author{
AlEXANDER DRANishniKov
}

\begin{abstract}
We prove the inequality
$\operatorname{asdim} A *_{C} B \leq \max \{\operatorname{asdim} A, \operatorname{asdim} B, \operatorname{asdim} C+1\}$.

Then we apply this inequality to show that the asymptotic dimension of any rightangled Coxeter group does not exceed the dimension of its Davis complex.
\end{abstract}

20F65, 20F55, 20F69

\section{Introduction}

Asymptotic dimension was introduced by Gromov as an invariant of finitely generated groups [20]. It is defined for metric spaces and applied to finitely generated groups with the word metric. Since by its definition it is quasi-isometry invariant, it does not depend on the choice of a finite generating set. It turns out that the asymptotic dimension is a coarse invariant in the sense of Roe [21]. Since all proper left invariant metrics on any countable group are coarsely equivalent (see Dranishnikov and Smith [16] and Shalom [22]), the notion of asymptotic dimension can be extended to all countable groups.

The interest in the asymptotic dimension was sparked by Gouliang Yu's proof of the Novikov Higher Signature conjecture for manifolds whose fundamental group has finite asymptotic dimension [23]. Similar progress on related conjectures was done under the assumption of finite asymptotic dimension in the works of Bartels [1], Carlsson and Goldfarb [9], the author [12] and the author with Ferry and Weinberger [13].

Finite asymptotic dimensionality is proven for many classes of groups. The exact computation of asymptotic dimension of groups is a more difficult task. To the best of my knowledge it is completed only for polycyclic groups and for hyperbolic groups. For polycyclic groups, the asymptotic dimension equals the Hirsch length: $\operatorname{asdim} \Gamma=h(\Gamma)$ (see Bell and Dranishnikov [4] for the inequality in one direction and Dranishnikov and Smith [16] for the other direction). The asymptotic dimension of a finitely generated hyperbolic group equals the covering dimension of its boundary plus one: 
$\operatorname{asdim} \Gamma=\operatorname{dim} \partial_{\infty} \Gamma+1$ by Buyalo [7] and Buyalo and Lebedeva [8]. In view of Bestvina-Mess' formula [6] we have asdim $\Gamma=\operatorname{vcd}(\Gamma)$ for those hyperbolic groups for which the virtual cohomological dimension is defined (eg for residually finite hyperbolic groups).

The Coxeter groups are considered a playground for many problems and conjectures in geometric group theory. All Coxeter groups have finite asymptotic dimension since they are isometrically embeddable in the finite product of trees by the author and Januszkiewicz [14]. The original such embedding is due to Januszkiewicz and it gives an estimate $\operatorname{asdim} \Gamma \leq|S|$ for a Coxeter system $(\Gamma, S)$. In [15] we noticed that the Januszkiewicz technique can be pushed to bring a better estimate to asdim $\Gamma \leq$ $\operatorname{ch}(N(\Gamma))$ where $N(\Gamma)$ is the nerve of $(\Gamma, S)$ and $\operatorname{ch}(N(\Gamma))$ is the chromatic number of the 1-skeleton of $N(\Gamma)$. A lower bound for the asymptotic dimension of Coxeter groups is given by Corollary 4.11 in [11]: $\operatorname{vcd} \Gamma \leq \operatorname{asdim} \Gamma$. Since $\operatorname{dim} N+1 \leq \operatorname{ch}(N)$ for every simplicial complex $N$ and $\operatorname{vcd}(\Gamma) \leq \operatorname{dim} \Sigma(\Gamma)=\operatorname{dim} N(\Gamma)+1$, it is natural to assume that $\operatorname{asdim} \Gamma \leq \operatorname{dim} N(\Gamma)+1$ where $\Sigma(\Gamma)$ denotes the Davis complex of $(\Gamma, S)$. In this paper we prove this inequality for right-angled Coxeter groups. Generally, the estimate asdim $\Gamma \leq \operatorname{dim} N(\Gamma)+1$ is not optimal. Perhaps the most natural guess would be that $\operatorname{asdim} \Gamma=\operatorname{vcd} \Gamma$, but in view of Bestvina's candidate among right-angled Coxeter groups for a counterexample to the Eilenberg-Ganea problem (see Davis [10]), this conjecture seems to be very difficult. The proposed equality has to be checked first for $\Gamma$ with 2-dimensional acyclic nerves $N(\Gamma)$.

In this paper the estimate asdim $\Gamma \leq \operatorname{dim} N(\Gamma)+1$ is proven by induction on dimension of the nerve. The main ingredient here is the inequality for the asymptotic dimension of the amalgamated product

$$
\operatorname{asdim} A *_{C} B \leq \max \{\operatorname{asdim} A, \operatorname{asdim} B, \operatorname{asdim} C+1\}
$$

which is proven in this paper.

This inequality was conjectured as an equality by Bell and the author [3], who proved it when $C$ is a finite group in [4]. Later J-P Caprice found an example among Coxeter groups where the inequality is strict.

As a corollary of $(*)$ this paper gives a new proof of the free product equality

$$
\operatorname{asdim} A * B=\max \{\operatorname{asdim} A, \operatorname{asdim} B, 1\} .
$$

The existing proof in Bell, Dranishnikov and Keesling [5] is quite long and it appeals to the asymptotic inductive dimension theory developed by the author and Zarichnyi [17].

The author would like to thank the NSF for support. 


\section{Asymptotic dimension}

We recall the definition of asymptotic dimension of a metric space [20]: $\operatorname{asdim} X \leq n$ if for every $r<\infty$ there exist uniformly bounded, $r$-disjoint families $\mathcal{U}^{0}, \ldots, \mathcal{U}^{n}$ of subsets of $X$ such that $\bigcup_{i} \mathcal{U}^{i}$ is a cover of $X$.

Let $r \in \mathbb{R}_{+}$be given and let $X$ be a metric space. We will say that a family $\mathcal{U}$ of subsets of $X$ is $r$-disjoint if $d\left(U, U^{\prime}\right) \geq r$ for every $U \neq U^{\prime}$ in $\mathcal{U}$. Here, $d\left(U, U^{\prime}\right)=$ $\inf \left\{d\left(x, x^{\prime}\right) \mid x \in U, x^{\prime} \in U^{\prime}\right\}$.

For a cover $\mathcal{U}$ of a metric space $X$ we denote by $L(\mathcal{U})=\inf _{U \in \mathcal{U}} \sup _{x \in X} d(x, X \backslash U)$ the Lebesgue number of $\mathcal{U}$. We recall that the order ord $\mathcal{U}$ of a cover $\mathcal{U}$ is the maximal number of elements with nonempty intersection.

We say that $(r, d)-\operatorname{dim} X \leq n$ if for every $r>0$ there exists a $d$-bounded cover $\mathcal{U}$ of $X$ with ord $\mathcal{U} \leq n+1$ and with the Lebesgue number $L(\mathcal{U})>r$. We refer to such a cover as to an $(r, d)$-cover of $X$.

Proposition 2.1 [3] For a metric space, asdim $X \leq n$ if and only if there is a function $d(r)$ such that $(r, d(r))-\operatorname{dim} X \leq n$ for all $r>0$.

Let $B_{R}(x)$ denote the closed $R$-ball centered at $x$ and let $N_{R}(A)=\{x \in X \mid d(x, A) \leq$ $R\}$ denotes the closed $R$-neighborhood of $A$. Thus, $B_{R}(x)=N_{R}(\{x\})$.

Proposition 2.2 Suppose that $X \subset Y$ is given the restricted metric and let $\mathcal{U}$ be an $(r, d)$-cover of $X$. Then $N_{r / 4}(X)$ admits an $(r / 4, d+r)$-cover $\tilde{\mathcal{U}}$ with ord $\tilde{\mathcal{U}} \leq \operatorname{ord} \mathcal{U}$.

Proof For every $U \in \mathcal{U}$ we define

$$
\bar{U}=\bigcup\left\{\operatorname{Int} B_{r / 2}(x) \mid d(x, X \backslash U) \geq r\right\} .
$$

Clearly, $N_{r / 2}(X) \subset \bigcup_{U \in \mathcal{U}} \bar{U}$. We show that $\operatorname{ord}\{\bar{U} \mid U \in \mathcal{U}\} \leq \operatorname{ord} \mathcal{U}$. Let $y \in$ $\bar{U}_{1} \cap \cdots \cap \bar{U}_{k}$. Let $x_{i} \in U_{i}$ be such that $d\left(x_{i}, y\right)<r / 2$ and $d\left(x_{i}, X \backslash U_{i}\right) \geq r$. Since $d\left(x_{i}, x_{1}\right)<r$ and $d\left(x_{i}, X \backslash U_{i}\right) \geq r$, it follows that $x_{1} \in U_{i}$ for all $i$. Thus, $U_{1} \cap \cdots \cap U_{k} \neq \varnothing$.

Let $\tilde{U}=\bar{U} \cap N_{r / 4}(X)$. Then $\tilde{\mathcal{U}}=\{\tilde{U}\}$ is an $(r / 4, d+r)$-cover with ord $\tilde{\mathcal{U}} \leq \operatorname{ord} \mathcal{U}$.

Let $K$ be a countable simplicial complex. There is a metric on $|K|$ called uniform which comes from the geometric realization of $K$. It is defined by embedding $K$ into the Hilbert space $\ell^{2}=\ell^{2}\left(K^{(0)}\right)$ by mapping each vertex $v \in K^{(0)}$ to a corresponding element of an orthonormal basis for $\ell^{2}$ and giving $K$ the metric it inherits as a subspace. 
A map $\varphi: X \rightarrow Y$ between metric spaces is uniformly cobounded if for every $R>0$, $\operatorname{diam}\left(\varphi^{-1}\left(B_{R}(y)\right)\right)$ is uniformly bounded. We call a map $\varphi: X \rightarrow|K|$ to a simplicial complex $c$-cobounded, $c \in \mathbb{R}_{+}$, if $\operatorname{diam}\left(\varphi^{-1}(\Delta)\right)<c$ for all simplices $\Delta \subset K$.

The following was proven by Gromov [20] (see also Bell and Dranishnikov [3] and Roe [21]).

Theorem 2.3 Let $X$ be a metric space. The following conditions are equivalent.

- $\operatorname{asdim} X \leq n$

- For every $\epsilon>0$ there is a uniformly cobounded, $\epsilon$-Lipschitz map $\varphi: X \rightarrow K$ to a uniform simplicial complex of dimension $n$.

This theorem is proved by using projections to the nerves of open covers. The projection $p_{\mathcal{U}}: X \rightarrow \operatorname{Nerve}(\mathcal{U}) \subset \ell^{2}(\mathcal{U})$ defined by the formula

$$
p_{\mathcal{U}}(x)=\left(\phi_{U}\right)_{U \in \mathcal{U}}, \quad \phi_{U}(x)=\frac{d(x, X \backslash U)}{\sum_{V \in \mathcal{U}} d(x, X \backslash V)}
$$

is called canonical.

A map $f: X \rightarrow Y$ between metric spaces is a coarse embedding if there exist nondecreasing functions $\rho_{1}$ and $\rho_{2}, \rho_{i}: \mathbb{R}_{+} \rightarrow \mathbb{R}_{+}$such that $\rho_{i} \rightarrow \infty$ and for every $x, x^{\prime} \in X$

$$
\rho_{1}\left(d_{X}\left(x, x^{\prime}\right)\right) \leq d_{Y}\left(f(x), f\left(x^{\prime}\right)\right) \leq \rho_{2}\left(d_{X}\left(x, x^{\prime}\right)\right) .
$$

Such a map is often called a coarsely uniform embedding or just a uniform embedding. The metric spaces $X$ and $Y$ are coarsely equivalent if there is a coarse embedding $f: X \rightarrow Y$ so that there is some $R$ such that $Y \subset N_{R}(f(X))$.

Observe that quasi-isometric spaces are coarsely equivalent with linear $\rho_{i}$.

Proposition 2.4 Let $f: X \rightarrow Y$ be a coarse equivalence. Then $\operatorname{asdim} X=\operatorname{asdim} Y$.

As a corollary we obtain that asdim $\Gamma$ is an invariant for finitely generated groups. One can extend this definition of asdim for all countable groups by considering left-invariant proper metrics on $\Gamma$. All such metrics are coarsely equivalent [16;22].

Theorem 2.5 [16] Let $G$ be a countable group. Then $\operatorname{asdim} G=\sup \operatorname{asdim} F$ where the supremum is taken over all finitely generated subgroups $F \subset G$.

For a subset $Y \subset X$ of a metric space $X$ when we write $\operatorname{asdim} Y$ we assume that $Y$ is taken with the metric obtained by restriction.

Also we use in this paper the following two theorems from [2]: 
Theorem 2.6 (Finite Union Theorem) For every metric space presented as a finite union $X=\bigcup X_{i}$ there is the formula

$$
\operatorname{asdim}\left(\bigcup X_{i}\right)=\max \left\{\operatorname{asdim} X_{i}\right\}
$$

Theorem 2.7 (Infinite Union Theorem) Let $X=\bigcup_{\alpha} X_{\alpha}$ be a metric space where the family $\left\{X_{\alpha}\right\}$ satisfies the inequality asdim $X_{\alpha} \leq n$ uniformly. Suppose further that for every $r$ there is a $Y_{r} \subset X$ with asdim $Y_{r} \leq n$ so that $d\left(X_{\alpha} \backslash Y_{r}, X_{\alpha^{\prime}} \backslash Y_{r}\right) \geq r$ whenever $X_{\alpha} \neq X_{\alpha^{\prime}}$. Then $\operatorname{asdim} X \leq n$.

We recall that the family $\left\{X_{\alpha}\right\}$ of subsets of $X$ satisfies the inequality asdim $X_{\alpha} \leq n$ uniformly if for every $r<\infty$ one can find a constant $R$ so that for every $\alpha$ there exist $r$-disjoint families $\mathcal{U}_{\alpha}^{0}, \ldots, \mathcal{U}_{\alpha}^{n}$ of $R$-bounded subsets of $X_{\alpha}$ covering $X_{\alpha}$.

The following Propositions are taken from [3, Proposition 2 and Lemma 1].

Proposition 2.8 For every simplicial map $g: X \rightarrow Y$ the mapping cylinder $M_{g}$ admits a triangulation with the set of vertices equal to the disjoint union of vertices of $X$ and $Y$.

We consider the uniform metric on $M_{g}$.

For a cover $\mathcal{U}$ of a metric space $X$ we denote by $b(\mathcal{U})=\sup _{U \in \mathcal{U}} \operatorname{diam}(U)$ the diameter of $\mathcal{U}$. We note that if for two covers $b(\mathcal{V})<L(\mathcal{U})$ then there is a map $G: \mathcal{V} \rightarrow \mathcal{U}$ with the property $G(V) \subset U$. Note that any such map $G: \mathcal{V} \rightarrow \mathcal{U}$ defines a simplicial map $g: \operatorname{Nerve}(\mathcal{V}) \rightarrow \operatorname{Nerve}(\mathcal{U})$ of the nerves.

We use the notation $\partial N_{r}(A)=\{x \mid d(x, A)=r\}$ for the boundary of the $r$-neighborhood and $r-\operatorname{Int}(A)=A \backslash N_{r}(X \backslash A)$ for the $r$-interior of $A$.

Lemma 2.9 [3] For every $n \in N$ there is a monotone function $\mu: \mathbb{R}_{+} \rightarrow \mathbb{R}_{+}$tending to infinity with the following property: Given $\epsilon>0$, let $W \subset X$ be a subset of a geodesic metric space $X$ and let $\lambda \geq 1 / \epsilon$. Then for every two covers $\mathcal{V}$ of $N_{\lambda}(\partial W)$ and $\mathcal{U}$ of $W$ by open subsets of $X$ with order $\leq n+1$, and with $L(\mathcal{U})>b(\mathcal{V})>L(\mathcal{V}) \geq \mu(\lambda)$, there is a $2 b(\mathcal{U})$-cobounded $\epsilon$-Lipschitz map $f: W \rightarrow M_{g}$ to the mapping cylinder of a simplicial map $g: \operatorname{Nerve}(\mathcal{V}) \rightarrow \operatorname{Nerve}(\mathcal{U})$ between the nerves such that $\left.f\right|_{\partial W}=\left.p_{\mathcal{V}}\right|_{\partial W}$ where $p_{\mathcal{V}}: N_{\lambda}(\partial W) \rightarrow \operatorname{Nerve}(\mathcal{V}) \subset M_{g}$ is the canonical projection.

We note that the formulation of Lemma 2.9 differs slightly from Lemma 1 in [3]. Namely, Lemma 2.9 becomes Lemma 1 if one considers the case $W=N_{r}(\partial W)$. Nevertheless the same formula for $f$ and the same proof as in [3] are valid for the general case. 
A partition of a metric space $X$ is a presentation as a union $X=\bigcup_{i} W_{i}$ such that $\operatorname{Int}\left(W_{i}\right) \cap \operatorname{Int}\left(W_{j}\right)=\varnothing$ whenever $i \neq j$.

Theorem 2.10 (Partition Theorem) Let $X$ be a geodesic metric space. Suppose that for every $R>0$ there is $d>0$ and a partition $X=\bigcup_{i}^{\infty} W_{i}$ with asdim $W_{i} \leq n$ uniformly in $i$ such that $(R, d)-\operatorname{dim}\left(\bigcup_{i} \partial W_{i}\right) \leq n-1$ where $\bigcup_{i} \partial W_{i}$ is taken with the metric restricted from $X$. Then asdim $X \leq n$.

Proof We apply Theorem 2.3. Given $\epsilon>0$ we construct a uniformly cobounded $\epsilon$-Lipschitz map $\phi: X \rightarrow K$. We apply the assumption with $R=4 \mu(1 / \epsilon)$ where $\mu$ is taken from Lemma 2.9. Let $\lambda=1 / \epsilon$. Since $\lambda \leq R / 4$ and $\mu(t) \geq t$, by Proposition 2.2 there is an $(r / 4,2 d)$-cover $\mathcal{V}$ of $N_{\lambda}\left(\bigcup_{i} \partial W_{i}\right)$ of order $\leq n$. We may assume that it is a cover by sets that are open in $X$. Let $\mathcal{V}_{i}=\left.\mathcal{V}\right|_{\partial W_{i}}$ be the restriction, ie, $\mathcal{V}_{i}$ consists of those elements of $\mathcal{V}$ that have a nonempty intersection with $\partial W_{i}$. Let $\mathcal{U}_{i}$ be a cover of $W_{i}$ with $L\left(\mathcal{U}_{i}\right)>2 d \geq b\left(\mathcal{V}_{i}\right)$ and with $b\left(\mathcal{U}_{i}\right)<D$ for all $i$ for some fixed $D$. By Lemma 2.9 there is a $2 D$-cobounded $\epsilon-$ Lipschitz map $f_{i}: W_{i} \rightarrow$ $M_{g_{i}}$ to a uniform complex where $M_{g_{i}}$ is the mapping cylinder of a simplicial map $g_{i}: \operatorname{Nerve}\left(\mathcal{V}_{i}\right) \rightarrow \operatorname{Nerve}\left(\mathcal{U}_{i}\right)$ and $f_{i}$ coincides on $\partial W_{i}$ with the canonical projection to the nerve $p_{\mathcal{V}}: \bigcup_{i} \partial W_{i} \rightarrow \operatorname{Nerve}(\mathcal{V})$. We define

$$
K=\left(\operatorname{Nerve}(\mathcal{V}) \coprod \coprod_{i} M_{g_{i}}\right) / \sim
$$

as the quotient space under identification along the complexes $\operatorname{Nerve}\left(\mathcal{V}_{i}\right)$. Then the union of $f_{i}$ defines a map $f: X \rightarrow K$. Clearly, $f$ is $2 D$-cobounded. Since $X$ is geodesic and each $f_{i}$ is $\epsilon$-Lipschitz, $f$ is $\epsilon$-Lipschitz with respect to the uniform metric on $K$.

\section{Asymptotic dimension of amalgamated product}

Let $A$ and $B$ be finitely generated groups and let $C$ be a common subgroup. We fix finite generating symmetric sets $S_{A}$ and $S_{B}$. Let $d$ denote the word metric on $A *_{C} B$ corresponding to the generating set $S_{A} \cup S_{B}$. The group $G=A *_{C} B$ acts on the Bass-Serre tree whose vertices are the left cosets $G / A \amalg G / B$ and the vertices $x A$ and $x B, x \in G$, and only them are joined by edges. The edges $[x A, x B]$ are labeled by the cosets $x C$. We consider the action of $G$ on the dual graph $K$. Thus vertices of $K$ are the left cosets $x C$. Two vertices $x C$ and $x^{\prime} C$ are joined by an edge if an only if the edges in the Bass-Serre tree with these labels have a common vertex. Note that $K$ is a tree-graded space in the sense of Drutu-Sapir [18] with pieces $\Delta(A)$ and $\Delta(B)$, the 1 -skeletons of the simplices spanned by $A / C$ or $B / C$. Thus, 
$K$ is partitioned into these pieces in a way that every two pieces have at most one common vertex and the nerve of the partition is a tree (see Figure 1). The graph $K$ has an additional property that all vertices are the intersection points of exactly two pieces of the different types $\Delta(A)$ and $\Delta(B)$. We consider the simplicial metric on $K$, ie, every edge has length one and we use the notation $|u, v|$ for the distance between vertices $u, v \in K^{(0)}$. For $u \in K^{(0)}$ by $|u|$ we denote the distance to the vertex with label $C$. Note $K$ has the unique geodesic property for every pair of vertices. There is a natural projection $\pi: G \rightarrow K$ defined by the action: $\pi(g)=g C$.

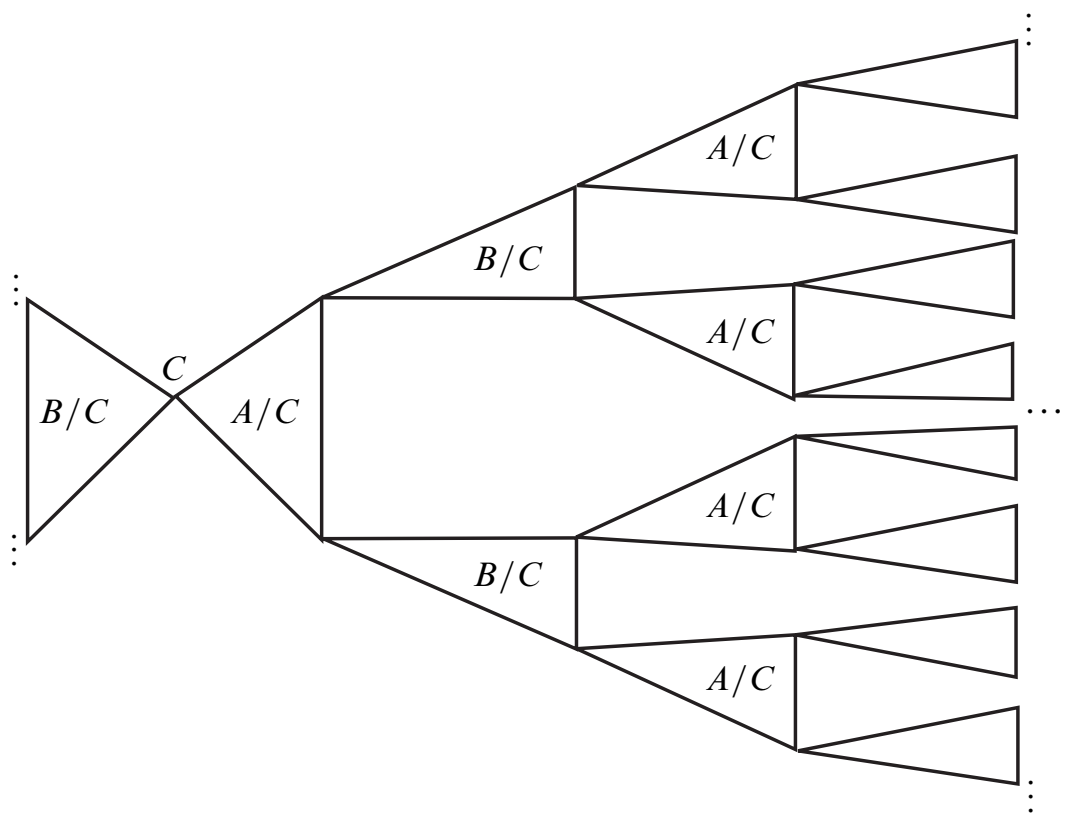

Figure 1

Assertion 3.1 The map $\pi: G \rightarrow K$ extends to a simplicial map of the Cayley graph of $G, \pi: C(G) \rightarrow K$.

Proof Let $g \in G$ and $s \in S_{A} \cup S_{B}$. If $s \in C$, then $\pi(g)=\pi(g s)$ and the edge $[g, g s] \subset C(G)$ is mapped to the vertex $g C=g s C$. With out loss of generality we may assume that $s \in A \backslash C$. We need to show that $\pi(g)=g C$ and $\pi(g s)=g s C$ are joined by an edge in $K$. Note $g A$ is the common vertex for the edges $[g B, g A]$ and $[g A, g s B]$ in the Bass-Serre tree. So the vertices corresponding these edges are joined by an edge in $K$. Thus the vertices $g C$ and $g_{s} C$ are joined by an edge in $K$.

As a corollary we obtain that $\pi$ is 1 -Lipschitz. 
The base vertex $C$ separates $K$ into two parts $K_{A} \backslash\{C\}$ and $K_{B} \backslash\{C\}$. Let $\bar{d}$ denote the graph metric on $K$. We denote by $B_{r}^{A}$ the $r$-ball, $r \in \mathbb{N}$, in $K_{A}$ centered at $C$. There is a partial order on vertices of $K$ defined as follows: $v \leq u$ if and only if $v$ lies in the geodesic segment $[C, u]$ joining the base vertex with $u$. For $u \in K^{(0)}$ of nonzero level and $r>0$ we denote by

$$
K^{u}=\left\{v \in K^{(0)} \mid v \geq u\right\}, \quad B_{r}^{u}=\left\{v \in K^{u}|| v|\leq| u \mid+r\right\} .
$$

For every vertex $u \in K^{(0)}$ represented by a coset $g C$ with $|u|$ even we have the equalities $B_{r}^{u}=g B_{r}^{A}, K^{u}=g K_{A}$ and hence the equalities $\pi^{-1}\left(B_{r}^{u}\right)=g \pi^{-1}\left(B_{r}^{A}\right)$ and $\pi^{-1}\left(K^{u}\right)=g \pi^{-1}\left(K_{A}\right)$.

We say that a set $F \subset G$ separates two subsets $H_{1}$ and $H_{2}$ in $G$ if it separates them in the Cayley graph $C(G)$, that is every path in $C(G)$ with the endpoints in $H_{1}$ and $\mathrm{H}_{2}$ meets $\mathrm{F}$.

Let $D_{R}=\{x \in G \mid d(x, C)=R\}$ be the boundary of the $R$-neighborhood of $C$ in $\pi^{-1}\left(K_{A}\right), R \in \mathbb{N}$. For $u \in K^{(0)}$ we denote $D_{R}^{u}=g_{u}\left(D_{R}\right), g_{u} \in G$, where the coset $g_{u} C$ represents $u$. Note that $\pi\left(D_{R}^{u}\right) \subset B_{R}^{u}$.

Proposition 3.2 For every vertex $u \in K$ with even $|u|$ and for every $v \in K^{(0)}$ incomparable with $u$ or satisfying $v<u$, the set $D_{R}^{u}$ separates $\pi^{-1}(v)$ and $\pi^{-1}\left(u^{\prime}\right)$ in $A *_{C} B$ whenever $u<u^{\prime}$ and $\left|u^{\prime}\right|-|u|>R$.

Proof Since $G$ acts by isometries, it suffices to show that $D_{R}$ separates $\pi^{-1}\left(K_{B}\right)$ and $\pi^{-1}\left(u^{\prime}\right)$ with $\left|u^{\prime}\right|>R$ and $u^{\prime} \in K_{A}$. In view of the fact that $\pi$ is 1 -Lipschitz, $d\left(C, \pi^{-1}\left(u^{\prime}\right)\right)>R$. Hence $D_{R}$ separates $C$ and $\pi^{-1}\left(u^{\prime}\right)$. By Assertion 3.1 the image of a path in $C(G)$ is a path in $K$. Since every path in $K$ from $K_{A}$ to $K_{B}$ hits the vertex $C$, it follows that every path in the Cayley graph from $\pi^{-1}\left(K_{A} \backslash C\right)$ to $\pi^{-1}\left(K_{B} \backslash C\right)$ hits the set $C=\pi^{-1}(\{C\})$. Hence every path from $\pi^{-1}\left(u^{\prime}\right)$ to $\pi^{-1}\left(K_{B}\right)$ hits $D_{R}$.

Proposition 3.3 If $R \leq r / 4$, then $d\left(g D_{R}, g^{\prime} D_{R}\right) \geq 2 R$ for $g, g^{\prime} \in G$ with $|g C|$, $\left|g^{\prime} C\right| \in n r, n \in \mathbb{N}$, and $g C \neq g^{\prime} C$.

Proof We use the notation $u=g C$ and $u^{\prime}=g^{\prime} C$ for the vertices in $K$. First consider the case when $|u| \neq\left|u^{\prime}\right|$. Since $\pi\left(g D_{R}\right) \subset B_{R}^{u}, \bar{d}\left(B_{R}^{u}, B_{R}^{u^{\prime}}\right) \geq r-R \geq 3 R$, and $\pi$ is 1 -Lipschitz, we obtain that $d\left(g D_{R}, g^{\prime} D_{R}\right) \geq 3 R$.

Let $|u|=\left|u^{\prime}\right|$ and let $x \in g D_{R}$ and $y \in g^{\prime} D_{R}$. Since every path in $K$ between $\pi(x)$ and $\pi(y)$ goes through the vertices $u$ and $u^{\prime}$ and a path in the Cayley graph $C(G)$ is projected to a path in $K$ (see Assertion 3.1), a geodesic from $x$ to $y$ in $C(G)$ passes through $g C$ and $g^{\prime} C$. Since $d(x, g C)=R$ and $d\left(y, g^{\prime} C\right)=R$ we obtain the inequality $d(x, y) \geq 2 R$. 
We fix two set-theoretic sections $s_{A}: A / C \rightarrow A$ and $s_{B}: B / C \rightarrow B$ of $\pi_{A}$ and $\pi_{B}$ and denote by $X=\operatorname{im}\left(s_{A}\right) \backslash C$ and $Y=\operatorname{im}\left(s_{B}\right) \backslash C$. These sections give rise the normal presentation of elements in $A *_{C} B$. Namely, every element $\gamma \in A *_{C} B$ can be presented uniquely in the following form $\gamma=z_{1} \ldots z_{k} c$ where $c \in C, z_{i} \in X \cup Y$, and $z_{i}$ are alternating in a sense that if $z_{i} \in X$ then $z_{i+1} \in Y$ and if $z_{i} \in Y$ then $z_{i+1} \in X$. Denote by $l\left(z_{1} \ldots z_{k} c\right)=k$ the length of the normal presentation. Clearly, $l(\gamma)=|\gamma C|$ where $\gamma C$ is treated as a vertex of $K$. If $X^{\prime}$ and $Y^{\prime}$ is a different choice of representatives and $\gamma=z_{1} \ldots z_{k} c$ and $\gamma=z_{1}^{\prime} \ldots z_{k}^{\prime} c^{\prime}$ are corresponding normal presentations, then $z_{1}^{\prime}=z_{1} c_{1}, z_{2}^{\prime}=c_{1}^{-1} z_{2} c_{2}, \ldots, z_{i}^{\prime}=c_{i-1}^{-1} z_{i} c_{i}, \ldots, z_{k-1}^{\prime}=c_{k-2}^{-1} z_{k-1} c_{k-1}$ and $z_{k}^{\prime} c^{\prime}=c_{k-1}^{-1} z_{k} c$ where $c_{i} \in C, i=1, \ldots, k$.

Assertion 3.4 Let $\gamma \in A *_{C} B$. Then $\|\gamma\| \geq d\left(\beta_{k} c, C\right)$ for the normal presentation $\gamma=\beta_{1} \ldots \beta_{k} c$ for any choice of representatives $X$ and $Y$.

Proof Let $\|\gamma\|$ be the word-length of $\gamma$ with respect to the generating set of $A *_{C} B$ and let $\gamma=t_{1} \ldots t_{n}, n=\|\gamma\|$, be a shortest presentation. Then the word $t_{1} \ldots t_{n}$ can be partitioned into a normal presentation (for some choice of $X$ and $Y$ ) $\gamma=\alpha_{1} \ldots \alpha_{k}$, $\alpha_{i} \in A$ (or $\alpha_{i} \in B$ ), with $k=l(\gamma)$. Then $\|\gamma\| \geq\left\|\alpha_{k}\right\|=d\left(\alpha_{k}, 1\right) \geq d\left(\alpha_{k}, C\right)$. If $X^{\prime}$ and $Y^{\prime}$ is a different choice of representatives and $\gamma=\beta_{1} \ldots \beta_{k} c$ are corresponding normal presentations, then $\beta_{1}=\alpha_{1} c_{1}, \beta_{2}=c_{1}^{-1} \alpha_{2} c_{2}, \ldots, \beta_{i}=c_{i-1}^{-1} \alpha_{i} c_{i}, \ldots$, $\beta_{k-1}=c_{k-2}^{-1} \alpha_{k-1} c_{k-1}$ and $\beta_{k} c^{\prime}=c_{k-1}^{-1} \alpha_{k}$ where $c_{i} \in C, i=1, \ldots, k$.

Thus, $\beta_{k} c^{\prime}=c \alpha_{k}$. This implies that $\|\gamma\| \geq d\left(\beta_{k} c, C\right)$.

Lemma 3.5 Let $\operatorname{asdim} A$, asdim $B \leq n$. Then $\operatorname{asdim}(A B)^{m} \leq n$ for all $m$ where $(A B)^{m}=A B \ldots A B \subset A *_{C} B$.

Proof We prove that asdim $A B \ldots A(B) \leq n$ by induction on the length of the product $k$. The inequality is a true statement for $k=1$. Assume that it holds for $k$. For the sake of concreteness assume that $k$ is odd. Thus, asdim $F_{1} \ldots F_{k} \leq n$ where $F_{2 i-1}=A$ and $F_{2 i}=B$. We show that asdim $F_{1} \ldots F_{k} B \leq n$. Consider the family $\{w B \mid l(w)=k\}$. Since all sets $w B$ are isometric to $B$, asdim $w B \leq n$ uniformly.

Given $r$ we define $Y_{r}=A B A B \ldots A B A C B_{r} \subset(A B)^{(k+1) / 2}=F_{1} \ldots F_{k+1}$ where $B_{r}$ is the $r$-ball in $B$. Note that $Y_{r}$ is quasi-isometric to the $k$-alternating product $A B \ldots A B A$ and hence asdim $Y_{r} \leq n$ by the induction assumption. We show that $d\left(w B \backslash Y_{r}, w^{\prime} B \backslash Y_{r}\right) \geq r$ whenever $w B$ is not equal to $w^{\prime} B$. Let $b, b^{\prime} \in B \backslash C B_{r}$. Then $d(b, C) \geq r$ and $d\left(b^{\prime}, C\right) \geq r$. Since $w^{-1} w^{\prime} \notin B$, the normal presentation of $b^{-1} w^{-1} w^{\prime} b^{\prime}$ ends with $c^{\prime} b^{\prime}$ for $c^{\prime} \in C$. Then by Assertion 3.4, $\left\|b^{-1} w^{-1} w^{\prime} b^{\prime}\right\| \geq$ $d\left(c^{\prime} b^{\prime}, C\right) \geq r$. Then by the Infinite Union Theorem (Theorem 2.7) we obtain that 
$\operatorname{asdim}\left(F_{1} \ldots F_{k} \cap L_{k}\right) B \leq n$ where $L_{k}$ is the set of all elements $w \in A *_{C} B$ with $l(w)=k, F_{i}=A(B)$ are alternating, and $F_{1}=A$. Let $L_{<k}$ be the set of all elements $w \in A *_{C} B$ with $l(w)<k$. The inequality $\operatorname{asdim}\left(F_{1} \ldots F_{m} \cap L_{<m}\right) B \leq n$ follows from induction assumption and the Finite Union Theorem (Theorem 2.6).

This Lemma first appeared in [2]. We present it here with a proof since the argument in [2] contains a gap in the proof of Proposition 3.

Theorem 3.6 For any finitely generated groups $A$ and $B$ and a common subgroup $C$ there is the inequality

$\operatorname{asdim} A *_{C} B \leq \max \{\operatorname{asdim} A, \operatorname{asdim} B, \operatorname{asdim} C+1\}$.

Proof Let $n=\max \{\operatorname{asdim} A, \operatorname{asdim} B, \operatorname{asdim} C+1\}$ and let $\pi: A *_{C} B \rightarrow K=$ $K_{A} \cup K_{B}$ be the projection to the graph dual to the Bass-Serre tree. In view of the Finite Union Theorem (Theorem 2.6) it suffices to show that $\operatorname{asdim} \pi^{-1}\left(K_{A}\right) \leq n$ and $\operatorname{asdim} \pi^{-1}\left(K_{B}\right) \leq n$. We prove the first.

We apply the Partition Theorem (Theorem 2.10). Let $R>0$ be given. Take $r>4 R$. In view of Proposition $3.2 G=X_{+} \cup X_{-}$with $X_{+} \cap X_{-}=D_{R}$ such that $X_{+} \subset$ $\pi^{-1}\left(K_{A}\right), \pi^{-1}\left(K_{B}\right) \subset X_{-}$and $D_{R}$ separates $X_{+} \backslash D_{R}$ and $X_{-} \backslash D_{R}$. For every vertex $u \in K_{A}$ we fix an element $g_{u} \in G$ such that the coset $g_{u} C$ represents $u$. We denote by $X_{ \pm}^{u}=g_{u}\left(X_{ \pm}\right)$and define $V_{r}=X_{+} \cap\left(\bigcap_{|u|=r} X_{-}^{u}\right)$. Note that $\pi\left(V_{r}\right) \subset B_{r+R}$. Let $V_{r}^{u}$ denote $g_{u}\left(V_{r}\right)$. Consider the partition

$$
\pi^{-1}\left(K_{A}\right)=\bigcup_{|u|=n r, n \in \mathbb{N}_{+}} V_{r}^{u} \cup N_{R}^{A}(C)
$$

where $N_{R}^{A}(C)=N_{R}(C) \cap \pi^{-1}\left(K_{A}\right)$.

Clearly, if $V_{r}^{u} \cap V_{r}^{w} \neq \varnothing$, then either $u<w$ and $|w|=|u|+r$ or $w<u$ and $|u|=|w|+r$. If $V_{r}^{u} \cap V_{r}^{w} \neq \varnothing$ and $u<w$ then $V_{r}^{u} \cap V_{r}^{w}=D_{R}^{w}$ where $D_{R}^{w}=g_{w} D_{R}$.

Thus

$$
Z=\bigcup_{|u|=n r, n \in \mathbb{N}_{+}} \partial V_{r}^{u}=\bigcup_{|u|=n r, n \in \mathbb{N}_{+}} D_{R}^{u} .
$$

We show that $(R, d)$-asdim $Z \leq n-1$ for some $d>0$. Since $D_{R}$ is coarsely equivalent to $C$, we have asdim $D_{R} \leq n-1$. Hence there is $d>0$ and an $(R, d)$-cover $\mathcal{U}$ of $D_{R}$ with ord $\mathcal{U} \leq n$. In view of Proposition 3.3, $\tilde{\mathcal{U}}=\bigcup_{|u|=n r, n \in \mathbb{N}_{+}} g_{u}(\mathcal{U})$ is an $(R, d)$-cover of $Z$.

Since $\pi^{-1}\left(B_{S}\right) \subset(A B)^{s+1}$, by Lemma 3.5 we have asdim $\pi^{-1}\left(B_{S}\right) \leq n$. Hence $\operatorname{asdim} \pi^{-1}\left(B_{r+R}\right) \leq n$ and so, $\operatorname{asdim} V_{r}^{u} \leq n$ uniformly. Note that $\operatorname{asdim} N_{R}^{A}(C) \leq n$ (in fact, it is $\leq n-1$ ). 
By the Partition Theorem (Theorem 2.10), $\operatorname{asdim} \pi^{-1}\left(K_{A}\right) \leq n$.

\section{Asymptotic dimension of right-angled Coxeter groups}

A symmetric matrix $M=\left(m_{s s^{\prime}}\right)_{s, s^{\prime} \in S}$ is called a Coxeter matrix if $m_{s s^{\prime}} \in \mathbb{N} \cup\{\infty\}$, and $m_{s s}=1$ for all $s \in S$. A group $\Gamma$ with a generating set $S$ is called a Coxeter group if there is a Coxeter matrix $M=\left(m_{s s^{\prime}}\right)_{s, s^{\prime} \in S}$ such that $\Gamma$ admits a presentation

$$
\left\langle S \mid\left(s s^{\prime}\right)^{m_{s s^{\prime}}}, s, s^{\prime} \in S\right\rangle .
$$

A Coxeter group $\Gamma$ is called even if all finite nondiagonal entries of $M$ are even. It is called right-angled if all finite nondiagonal entries equal 2.

Every subset $W \subset S$ defines a subgroup $\Gamma_{W} \subset \Gamma$ which will be called parabolic. Let $(\Gamma, S)$ be a Coxeter group with a generating set $S$ and with a presentation given by means of a Coxeter $S \times S$ matrix $M$. The nerve $N(\Gamma)$ is a simplicial complex with the set of vertices $S$ where a subset $W \subset S$ spans a simplex if and only if the group $\Gamma_{W}$ is finite. Thus, $s, s^{\prime} \in S, s \neq s^{\prime}$, form an edge if and only if $m_{s s^{\prime}} \neq \infty$. We call the number $m_{s s^{\prime}}$ a label of the edge $\left[s s^{\prime}\right]$. By $N^{\prime}$ we denote the barycentric subdivision of $N$. The cone $C=$ Cone $N^{\prime}$ over $N^{\prime}$ is called a chamber for $\Gamma$. The Davis complex $\Sigma=\Sigma(\Gamma, S)$ is the image of a simplicial map $q: \Gamma \times C \rightarrow \Sigma$ defined by the following equivalence relation on the vertices: $a \times v_{\sigma} \sim b \times v_{\sigma}$ provided $a^{-1} b \in \Gamma_{\sigma}$ where $\sigma$ is a simplex in $N$ and $v_{\sigma}$ is the barycenter of $\sigma$. We identify $C$ with the image $q(e \times C)$. The group $\Gamma$ acts properly and simplicially on $\Sigma$ with the orbit space equivalent to the chamber. Thus, the Davis complex is obtained by gluing the chambers $\gamma C, \gamma \in \Gamma$ along their boundaries. The main feature of $\Sigma$ is that it is contractible (see Davis [10]).

Theorem 4.1 For every right-angled Coxeter group $\operatorname{asdim} \Gamma \leq \operatorname{dim} N(\Gamma)+1$.

Proof We prove this inequality by induction on the dimension of the nerve $N=$ $N(\Gamma)$. If $\operatorname{dim} N=0$, then $\Gamma$ is virtually free group (possibly of zero rank) and hence $\operatorname{asdim} \Gamma \leq 1=\operatorname{dim} N+1$.

Let $\operatorname{dim} N=n$ and let $N$ be finite. We prove the inequality asdim $\Gamma \leq n+1$ by induction on the number of vertices in $N$. If this number is minimal, ie, $n+1$, the inequality holds since the group $\Gamma$ in this case is finite. We assume that there is a vertex $v \in N$ such that the $\operatorname{star} \operatorname{st}(v, N)$ does not contain all other vertices of $N$. If there is no such $v$, then the 1-dimensional skeleton $N^{(1)}$ coincides with the 1-skeleton of a simplex $\Delta$. Since the group is right-angled, $N=\Delta$, and the group is finite, so this case has been already considered. We take $K$ to be the link $\operatorname{Lk}(v, N)$ of such vertex 
$v$ and take $N_{1}$ to be the star st $(v, N)$ of this vertex. We define $N_{2}=N \backslash \operatorname{Ost}(v, N)$ where $\operatorname{Ost}(v, N)$ is the open star of $v$.

Then $\Gamma=\Gamma_{N_{1}} * \Gamma_{K} \Gamma_{N_{2}}$. By the induction assumption, asdim $\Gamma_{K} \leq n$. By the internal induction, asdim $\Gamma_{N_{i}} \leq n+1, i=1,2$. Then Theorem 3.6 implies asdim $\Gamma \leq n+1$.

Corollary 4.2 For every right-angled Coxeter group $\operatorname{asdim} \Gamma \leq \operatorname{dim} \Sigma(\Gamma)$ where $\Sigma(\Gamma)$ is the Davis complex.

In view of recent result of Dymara and Schick [19] we obtain:

Corollary 4.3 For a right-angled building $X$, asdim $X \leq \operatorname{dim} X$.

Remark In order to extend Theorem 4.1 to all Coxeter groups one needs to show the inequality $\operatorname{asdim} \Gamma \leq \operatorname{dim} N+1$ in the case when $N^{(1)}$ is the 1 -skeleton of a simplex.

\section{References}

[1] A C Bartels, Squeezing and higher algebraic K-theory, K-Theory 28 (2003) 19-37 MR1988817

[2] G Bell, A Dranishnikov, On asymptotic dimension of groups, Algebr. Geom. Topol. 1 (2001) 57-71 MR1808331

[3] G Bell, A Dranishnikov, On asymptotic dimension of groups acting on trees, Geom. Dedicata 103 (2004) 89-101 MR2034954

[4] G C Bell, A N Dranishnikov, A Hurewicz-type theorem for asymptotic dimension and applications to geometric group theory, Trans. Amer. Math. Soc. 358 (2006) 4749-4764 MR2231870

[5] G C Bell, A N Dranishnikov, J E Keesling, On a formula for the asymptotic dimension of free products, Fund. Math. 183 (2004) 39-45 MR2098148

[6] M Bestvina, G Mess, The boundary of negatively curved groups, J. Amer. Math. Soc. 4 (1991) 469-481 MR1096169

[7] S V Buyalo, Asymptotic dimension of a hyperbolic space and the capacity dimension of its boundary at infinity, Algebra i Analiz 17 (2005) 70-95 MR2159584; translation in St. Petersburg Math. J. 17 (2006) 267-283

[8] S V Buyalo, ND Lebedeva, Dimensions of locally and asymptotically self-similar spaces, Algebra i Analiz 19 (2007) 60-92 MR2319510

[9] G Carlsson, B Goldfarb, On homological coherence of discrete groups, J. Algebra 276 (2004) 502-514 MR2058455 
[10] M W Davis, The geometry and topology of Coxeter groups, London Math. Soc. Monographs Ser. 32, Princeton University Press (2008) MR2360474

[11] A Dranishnikov, Cohomological approach to asymptotic dimension arXiv: math.MG/0608215

[12] A Dranishnikov, On hypersphericity of manifolds with finite asymptotic dimension, Trans. Amer. Math. Soc. 355 (2003) 155-167 MR1928082

[13] A Dranishnikov, S C Ferry, S A Weinberger, An etale approach to the Novikov conjecture, Comm. Pure Appl. Math. 61 (2008) 139-155 MR2368371

[14] A Dranishnikov, T Januszkiewicz, Every Coxeter group acts amenably on a compact space, from: "Proceedings of the 1999 Topology and Dynamics Conference (Salt Lake City, UT)”, Topology Proc. 24 (1999) 135-141 MR1802681

[15] A Dranishnikov, V Schroeder, Embedding of Coxeter groups in a product of trees arXiv:math.GR/0402398

[16] A Dranishnikov, J Smith, Asymptotic dimension of discrete groups, Fund. Math. 189 (2006) 27-34 MR2213160

[17] A Dranishnikov, M Zarichnyi, Universal spaces for asymptotic dimension, Topology Appl. 140 (2004) 203-225 MR2074917

[18] C Druţu, M Sapir, Tree-graded spaces and asymptotic cones of groups, Topology 44 (2005) 959-1058 MR2153979 With an appendix by Denis Osin and Sapir

[19] J Dymara, T Schick, Buildings have finite asymptotic dimension arXiv: math.MG/070399v1

[20] M Gromov, Asymptotic invariants of infinite groups, from: "Geometric group theory, Vol. 2 (Sussex, 1991)”, London Math. Soc. Lecture Note Ser. 182, Cambridge Univ. Press (1993) 1-295 MR1253544

[21] J Roe, Coarse cohomology and index theory on complete Riemannian manifolds, Mem. Amer. Math. Soc. 104 (1993) x+90 MR1147350

[22] Y Shalom, Harmonic analysis, cohomology, and the large-scale geometry of amenable groups, Acta Math. 192 (2004) 119-185 MR2096453

[23] G Yu, The Novikov conjecture for groups with finite asymptotic dimension, Ann. of Math. (2) 147 (1998) 325-355 MR1626745

University of Florida, Department of Mathematics

PO Box 118105, 358 Little Hall, Gainesville, FL 32611-8105, USA

dranish@math.ufl.edu

http://www.math.ufl.edu/ dranish

Received: 17 May 2007 Revised: 13 February 2008 